\title{
INGENIERÍA EN COMUNICACIÓN SOCIAL DE LA EXPERIENCIA ESTÉTICA DEL ROCK Y EL JAZZ. Explorando la configuración de la vida contemporánea a través de la música \\ ENGINEERING IN SOCIAL COMMUNICATION OF AESTHETIC EXPERIENCE OF ROCK AND JAZZ. Exploring the configuration of contemporary life through music
}

Jesús Galindo Cáceres*

\section{RESUMEN}

El texto está dividido en cinco partes. La primera se dedica a una definición constructiva de la estética y su relación con la vida social. Estética entendida como la articulación de la vida cotidiana con lo múltiple y diverso, en la figura de la experiencia sensible-cognitiva de lo particular en el contexto de la complejidad. La segunda presenta en forma sintética un esquema de trabajo de la Ingeniería en Comunicación Social. No todo el modelo, sólo una síntesis con el énfasis en la configuración de los sistemas de comunicación, las formas concretas de la vida social en las situaciones cotidianas del presente, articulando sistemas de información, predeterminaciones que vienen del pasado.

La tercera parte explora la relación entre la estética presentada en el primer punto y la Ingeniería en Comunicación Social presentada en el segundo punto. La experiencia estética se vive en el presente, es una forma de los sistemas de comunicación. Depende de la configuración de las situaciones vivibles y vividas, la forma y fuerza de la experiencia estética.

La cuarta parte propone un esquema sintético del desarrollo del rock y del jazz en el mundo contemporáneo, en particular en México. El rock ha tenido una presencia más popular por ser más simple y directo, el jazz es más complejo, más denso en la experiencia estética. En México el rock ha tenido una presencia que se agota en mucho en una situación de excitación extrema y contestataria simple. El jazz no se ha desarrollado en México al requerir una formación y articulación compleja con la vida social.

En la quinta parte del artículo se presenta la tesis de las diferencias constructivas de la estética del jazz y del rock, la primera más compleja y articuladora, la segunda más simple y desarticuladora. El jazz complejiza la vida social, el rock la simplifica. El jazz requiere de una atención y de una empatía compleja con la situación musical y con el contexto que articula. El rock requiere una atención mínima, su empatía es simple y en ocasiones no es necesaria, es sólo consumo individual. Son formas de música distintas, son formas de vivencia en vivo distintas, son formas de construcción del sentido y de empatía distintas.

Palabras clave: experiencia estética, ingeniería en comunicación social, Rock, Jazz, vida contemporánea.

\section{SUMMARY}

The text is divided into five parts. The first one is devoted to a constructive definition of aesthetics and its relation to social life. Aesthetics understood as the articulation of everyday life with multiple and diverse, in the figure of the sensible-cognitive experience of the particular in the context of complexity. The second one presents in summary form a roadmap of Engineering in Social Communication. Not whole model, only a summary with the emphasis on the configuration of the communication systems, the concrete forms of social life in everyday situations in the present, linking information systems, presets that come from the past.

The third part explores the relationship between aesthetics presented at the first point and Engineering in Social Communication presented in the second point. The aesthetic experience is lived in the present, it is a form of communication systems. It depends on the configuration of situations livable and lived the shape and strength of the aesthetic experience.

The fourth part proposes a synthetic scheme for the development of rock and jazz in the contemporary world, particularly in Mexico. The rock has been a presence more popular because is more simple and direct, jazz is more complex, more dense in the aesthetic experience. In Mexico the rock has had a presence that was very exhausted in a situation of extreme excitement and rebellious simple. Jazz has not developed in Mexico by requiring a complex formation, and a complex joint with social life.

In the fifth part of the article is presented the thesis of the constructive differences of aesthetics of jazz and rock, the first one more complex and articulator, the second one more simple and kilter. Jazz makes more complex social life, rock more simple it. Jazz requires of a complex care and empathy with the musical situation and with the context that articulates it. The rock requires minimal attention, empathy is simple and sometimes it is not needed, it is only individual consumption. They are different forms of music are different forms of experience in live, are ways of making sense and empathy different.into society of learning and intelligence.

Keywords: aesthetic experience, Engineering in Social Communication, Rock, Jazz, contemporary life. 
1. La Experiencia Estética y la Vida Social

La propuesta general aquí, es un apunte de definición constructiva de la estética en su relación con la vida social. Estética entendida como la articulación de la vida cotidiana con lo múltiple y lo diverso, en la figura de la experiencia sensible-cognitiva de lo particular en el contexto de la complejidad. Parece mucho, lo es, vamos explorando por partes lo que desde una Ingeniería Social se puede entender por estética.

El punto clave es la relación entre la situación de la experiencia y el movimiento general de nuestra vida. La estética es entendida aquí en principio como una experiencia, como algo que le sucede a una percepción cognitiva y que la modifica en su configuración y relación estratégica con el mundo reconstruido por lo acontecido. Una vez que esto sucede, como un detonador de un nivel de composición y organización superior de la percepción y la vivencia cotidiana, el movimiento de la vida es afectado, y a partir de entonces aparece la posibilidad de profundizar y sustentar a largo plazo lo sucedido, o que aquello vaya desapareciendo en el tiempo. Para una y otra trayectoria se requiere de un contexto y de una práctica que refuerce o que diluya lo emergente.

La experiencia estética sucede, y este es un fenómeno extraordinario, pero una vez que sucede lo que sigue es que se mantenga y desarrolle la reconfiguración obtenida, o que esa reconfiguración desaparezca poco a poco. Para una y otra cosa debe pasar algo que lo promueva. $Y$ ese algo es el asunto que liga todo esto con el rock y el jazz como tecnologías sociales de la experiencia estética.

En primer lugar está el fenómeno del acontecimiento de la experiencia estética. ¿Qué sucede? ¿Cómo sucede? ¿Cuándo sucede? La vida social es la mayor parte del tiempo lineal, una configuración de usos y costumbres, de rutinas, de escenas que se repiten una y otra vez. En el mejor de los casos esto es funcional, permite que el orden y la organización de las acciones e interacciones sucedan con un mínimo de conflicto en el marco de programas trazados por las Ingenierías Sociales previas, y todo acontece y el mundo es estable. En otros casos, muchos, sucede lo contrario, los programas no están protegidos ni estables, las trayectorias de la vida viven un estado de degradación constante, con gran gasto de energía, con niveles de conflicto y con una creciente angustia $\mathrm{y}$ ansiedad.
Desde la perspectiva general de la Ingeniería Social lo que agita la vida y la renueva desde un punto de vista energético y vital es la excitación, que requieren situaciones que despierten y pongan en alerta a los organismos para mantener en ellos un mínimo de funcionalidad; lo contrario, el aburrimiento, degrada a los organismos en su relación ecológica con los demás y el desarrollo de sí mismo. Así que la excitación es indispensable, de ahí el deporte, el juego, la sensación de aventura, el cortejo. Hay un nivel básico, simple, de la excitación, la sensación de despertar, de alerta. Pero también hay niveles superiores, complejos, cuando la ambición de sentido y la imaginación crecen y requieren de cierta estimulación cognitiva más rica y sofisticada. Las dos configuraciones de excitación pueden presentarse como alternas, como opciones únicas, o como opciones complementarias. En el segundo tipo está lo que nombramos como arte, y en su campo se ubica la música.

La música y otras prácticas de las llamadas artísticas están cargadas de complejidad cognitiva, tienen una parte asociada a la vivencia más simple, y tienen un desarrollo que les otorga un status de vivencias complejas. La música y otras formas de arte colaboran con la vida funcional o disfuncional del orden social y sus programas, en donde la excitación es parte de lo necesario, pero también forman parte de la discontinuidad de la complejidad, son tecnologías de la experiencia estética.

Detengámonos en estas imágenes un momento, una vida lineal funcional o no, y la vivencia de la excitación y la experiencia estética, un cuadro sintético de construcción de la vida social. La excitación es fundamental, común, normal, la diferencia entre la decadencia sistémica o la regeneración vital. La experiencia estética es escasa, extraordinaria, posible, reordena la percepción y el programa de articulación individuo-mundo hacia horizontes de mayor sensibilidad y necesidad. Están asociadas en el corazón de la vivencia, la emoción. Y a partir de ahí pueden incluso parecerse en ciertos momentos, en ciertos puntos, pero también se separan y diferencian en otros hasta ser casi por completo distintas.

Todos los seres vivos conocemos y vivimos la vivencia de la excitación, tiene una base biológica, natural, se requiere de una Ingeniería Social especializada para preservarla y promoverla. La experiencia estética es una configuración que se desprende de la biología y procesa otro nivel de la vida humana y social, para vivirla y cuidarla se requiere otra Ingeniería Social especializada y colaboradora de la anterior. La 
excitación es natural y aun así necesita un cuidado social. La experiencia estética también puede presentarse en forma casi natural, y requiere mayor cuidado y atención especial que la excitación.

Uno de los temas de mayor interés y de gran dificultad para la Ingeniería Social es la respuesta a ¿cómo sucede la experiencia estética? Imaginemos por un momento una situación en donde una persona frente a una obra plástica siente algo, y de pronto se transforma, como si fuera otra persona por un momento, viendo más, imaginando más, necesitando más información, ligado a más cosas y personas. Vive en ese momento un trance hacia una experiencia estética, y le gusta, no sabe bien cómo sucedió, no sabe cómo puede volver a suceder, quizás hasta le asusta un poco lo acontecido. Lo cierto es que la vida no será igual después de esa vivencia que se construye en cierta experiencia de algo más y mejor que lo ordinario.

Para la Ingeniería Social el tema es cómo conocer qué sucedió con la mayor precisión posible, y cómo gestionar que suceda, dado que construye personas más complejas y eso es deseable en cierto marco constructivo. La vivencia estética puede ser promovida, inducida. Tanto así que los individuos por sí mismos también tienen la experiencia de su gestión. Por ejemplo, después de escuchar algo que lo ha movido de fondo, buscan música similar para volver a tener la misma vivencia. Es posible afirmar que el arte se ha desarrollado en forma social en buena parte debido a estas vivencias y experiencias.

Aquí la lectura general es que el rock en principio provee de una vivencia de excitación que rompe la linealidad de la vida cotidiana, y se posiciona como un fenómeno social de gran importancia por esta configuración. El jazz tiene en su raíz también la misma situación, pero sucede que en su desarrollo la trasciende, la experiencia estética está más cerca del jazz que del rock, y la excitación es parte de ambas vivencias, pero parece más concentrada en el rock. A partir de aquí el tema es de exploración, hay formas de rock que se mueven a un registro más estético, y hay formas del jazz que se concentran más en registro de la excitación. El punto es que uno está más cerca en general de un registro que del otro, y eso es muy interesante, razón suficiente para que la Ingeniería Social haya desarrollado en ambos campos diversas formas tecnológicas, y a su vez haya posibilitado una gran inversión de energía y recursos en su posicionamiento como fenómenos sociales y culturales en el mundo contemporáneo.

\section{Ingeniería en comunicación social}

En este segundo punto se trata de presentar en forma sintética un esquema de trabajo de la Ingeniería en Comunicación Social. No se presenta todo el modelo, sólo una selección sintética con el énfasis en la configuración de los sistemas de comunicación, las formas concretas de la vida social en las situaciones cotidianas del presente, articulando sistemas de información, predeterminaciones que vienen del pasado. La propuesta de Ingeniería en Comunicación Social es la asociación entre una Ingeniería Social General y un apunte de Comunicología Social, entre la perspectiva general de construcción de la vida social a partir de la aplicación y cuidado de tecnologías sociales específicas, y la visión conceptual constructiva de una ciencia de la comunicación social.

La base para imaginar una Ingeniería Social de cualquier tipo es la Cultura de Investigación, en particular la que tiene su asentamiento en una cultura científica. La ciencia nos provee de dos configuraciones que nos empoderan ante el mundo, un sentido enriquecido de las cosas que observamos, y un método para construir ese sentido a partir de la observación sistematizada. Todo parte de la observación.

La información y la observación van de la mano, la información es el objeto de pensamiento en que la observación científica se concentra. $Y$ de ahí se distinguen otras operaciones tales como el registro de lo observado, la descripción de lo observado, la sistematización de lo observado, el análisis de lo observado, la organización de lo observado y la síntesis en conocimiento de lo observado. Esta es una configuración de operaciones sobre las cuales se construye un programa metodológico que inicia su camino observando algo, y lo culmina sintetizando conocimiento sobre ese algo observado. 
El método trabaja sobre información observada, la metodología y la tecnología de investigación trabajan sobre información observada. La cultura de investigación se compone de las formas en como configuramos nuestra operación básica de la observación para desarrollarla hasta sintetizar conocimiento, construcción de sentido enriquecido sobre algo. Ese sentido sobre algo siempre es una configuración que parte del mundo de la acción y regresa al mundo de la acción con más recursos, conocimiento, para poder actuar en él. Este es el punto clave del discurso que asocia a la ciencia con la ingeniería, y por tanto a la ciencia de lo social y a la ingeniería de lo social, a la Ciencia de la Comunicación Social y a la Ingeniería en Comunicación Social.

El proyecto de Ingeniería en Comunicación Social es un proyecto que apunta hacia una Sociogenética de la comunicación social. Vivimos en configuraciones sociales que pueden ser descritas y entendidas desde un espacio conceptual de la comunicación, desde esta perspectiva somos configuraciones de comunicación social y a través de ellas convivimos, nos asociamos, luchamos, colaboramos, competimos, construimos y destruimos. Es posible entonces un proyecto y un programa de trabajo científico que permita observar cómo sucede esto, con todas sus implicaciones y variaciones. $Y$ si esto es posible gracias a una propuesta científica sobre la comunicación social, la Comunicología, también es posible aprender de este sentido enriquecido para hacer lo mismo con la ventaja del conocimiento sobre ello, o intervenirlo para modificar en alguna dirección lo que hacemos para obtener ventajas en ello de diverso tipo, lo cual es el espacio constructivo de una Ingeniería en Comunicación Social.

En este apunte aparecen dos componentes que requieren un comentario más por el momento. La matriz que permite entender a la vida social como comunicación, desde la comunicación, construida como figura de comunicación. Y la matriz que permite pretender hacer algo con ese conocimiento. En un caso estamos ante la pregunta por la posibilidad y la intención constructiva de una ciencia de la comunicación, la Comunicología, y en el segundo caso estamos ante la pregunta por la posibilidad y la intención constructiva de una Ingeniería Social desde la comunicación, la Ingeniería en Comunicación Social.

Los conceptos centrales en la Comunicología son el sistema de información y el sistema de comunicación. Los sistemas de información representan en la propuesta teórica a la prescripción, a lo que en otros contextos conceptuales se nombra como código. Los sistemas de información están detrás de todo comportamiento ordenando la secuencia de las acciones y los sentidos que las significan, son el pasado ordenado que prescribe, ordena, prohíbe, indica, enfoca.

El mundo social puede ser percibido como una matriz de sistemas de información en operación, lo que está detrás de toda acción. Por otra parte están los sistemas de comunicación. Si los sistemas de información son un vector que viene del pasado hacia el presente, los sistemas de comunicación son un vector que mueve el presente hacia futuros posibles. Parten del presente, en el juego, interacción, conflicto, lucha, negociación, entre los diversos sistemas de información.

Los actores sociales están determinados del pasado al presente por los sistemas de información, y en su acción presente los mueven dentro de los sistemas de comunicación hacia escenarios de acción posible. En tanto que los sistemas de información ordenen a los sistemas de comunicación, el mundo se reproduce en forma similar, pero en el escenario contrario, los sistemas de comunicación desconfiguran, alteran, modifican, diluyen, transforman a los sistemas de información. El presente condicionado por el pasado reproduce a los sistemas de información que prescriben al comportamiento, el presente liberado del pasado altera la prescripción y lleva a nuevos escenarios de orden y forma sociales, son los sistemas de comunicación permitiendo que diversos sistemas de información se alteren unos a otros, o debilitando la realización de ellos por la fuerza de la novedad en las situaciones presentes que olvidan a los sistemas de información, los alteran, los reconfiguran.

La Ingeniería en Comunicación parte de esta concepción comunicológica de la vida social y la lleva al escenario de la construcción voluntaria posible. El ingeniero puede administrar diseños previos, puede hacerles ajustes, y puede hacer nuevos diseños. El proyecto de Ingeniería en Comunicación Social en general realiza primero diagnóstico de las situaciones presentes, para identificar el status de los sistemas de información y los sistemas de comunicación, y con ello propone acciones en la forma de tecnologías sociales estandarizadas que ya forman parte de los sistemas de información social culturales, y con ello interviene en las trayectorias sociales reforzando tendencias, incrementándolas, diluyéndolas, o sólo acompañándolas. 
La Ingeniería en Comunicación Social puede percibir al rock y al jazz como tecnologías sociales que le hacen algo a la gente, y sintetizar en consecuencia estratégicas en el sentido de reforzar, modificar, acompañar. Pero primero tiene que entender lo que pasa, y ahí la Comunicología es el gran instrumento de percepción y diagnóstico.

\section{Ingeniería en comunicación social y experiencia estética}

Esta tercera parte explora la relación entre la experiencia estética presentada en el primer punto y la Ingeniería en Comunicación Social presentada en el segundo punto. La experiencia estética parte de la vivencia en el presente, es una forma de los sistemas de comunicación. Depende de la configuración de las situaciones vivibles la forma y fuerza de la vivencia estética, depende en primer lugar de las condiciones de posibilidad que el contexto y la historia prescriben, los sistemas de información. En cierto sentido la vivencia estética es una de las formas máximas de configuración de los sistemas de comunicación, en ella los sistemas de información prescritos pueden ser reconfigurados por completo. De ahí la importancia de comprenderla y de aprender a promoverla. De la vivencia estética que da forma a la experiencia estética puede depender la reactivación de todo un sistema social que se vuelve pesadamente prescriptivo, agotando posibilidades de cambio y de regeneración vital.

Los sistemas de comunicación son la configuración del presente en la Comunicología social general. El pasado, los sistemas de información, busca reproducirse en el presente a través de los usos y costumbres y todo el blindaje que la propia vida social y su ingeniería ha diseñado para ello. Si el pasado se reproduce los sistemas de comunicación son una ejecución del programa de los sistemas de información, la vida no cambia, el presente duplica al pasado. Pero si algo sucede en el presente, si los sistemas de comunicación se activan en un sentido situacional frente a la prescripción de los sistemas de información, entonces el cambio acontece, la vida se transforma, los sistemas de información no se reproducen, son modificados por algo que pasó en el presente, en los sistemas de comunicación, el presente inaugura un futuro distinto al pasado.

Este tipo de mutación acontece todo el tiempo, en aspectos pequeños de la vida social cotidiana, en aspectos mayores de la vida social. El cómo sucede esto es parte sustantiva del trabajo de observación de la Ingeniería en Comunicación Social. Conocer las formas en que el cambio se produce o no, puede promover el que se pueda incidir en ese metabolismo a partir de diseños hechos para tales condiciones, modificando o no a voluntad, y según un diseño lo que acontece. La historia de la Ingeniería Social está plagada de ejemplos de esta intención, muchos muy exitosos, otros no tanto, y muchos fracasos. El punto es cómo es que el presente, el sistema de comunicación en donde se encuentran los diversos sistemas de información en situación, reproduce al pasado, o cómo es que el presente inicia una nueva configuración hacia el futuro.

Ejemplo. En el caso de la vida familiar. Una niña crece dentro de un programa que prescribe comportamientos y sentidos según cada etapa en el ciclo de vida. Todo ello está cifrado en sistemas de información codificados, la vida de la niña como si fuera un guion que está escrito de ante mano y que la niña ira incorporando y ejecutando según la eficiencia de su puesta en forma.

Si todo sale conforme al programa, la vida de la niña será tal cual está previsto en los sistemas de información diseñados para ello. Para que eso suceda se necesita que haya un control estratégico sobre la ecología situacional de la niña, que lo que se requiere para que el programa se ejecute aparezca en el orden y la configuración necesarios. Esto no suele suceder así del todo, siempre hay imprevistos, errores en la programación y en la ejecución, variables ambientales fuera de control, y así diciendo. Todo ello es el mundo del presente, el sistema de comunicación social. Este está caracterizado por la interacción de los sistemas de información en situación. Por una parte la prescripción de los sistemas de información del pasado hacia el presente, y por otra parte la interacción de los sistemas de información entre sí en el presente.

La primera configuración reproduce al pasado en el presente y prescribe al futuro en el mismo sentido. La segunda configuración posibilita mutuas afectaciones entre los diversos sistemas de información compitiendo por prescribir, el resultado puede ser un reordenamiento de la jerarquización prescriptiva de los sistemas de información, o una reordenamiento interno de los sistemas información por el efecto de la interacción con otros. Como sea el presente puede y lo hace, modificar al pasado, reconfigurando la relación entre sistemas de información y el interior de los sistemas de información mismos, hacia el futuro. 
La niña interactúa con otras niñas y con mundos y visiones distintas a través de los medios de difusión de información y el internet, el programa de su vida prescrito por su religión, su cultura regional, su clase social, su familia, se ve intervenido por todas estas variables exteriores a la programación básica. La niña vive entonces una vida distinta a la que estaba programada, su vivencia situacional dentro de un sistema de comunicación más amplio y rico del que se suponía modifica la reproducción del pasado e inaugura en el presente mutante futuros posibles.

La excitación forma parte de los programas sociales inscritos en los sistema de información, y puede moldearse y ecualizarse según esos programas. Pero también sucede que lo que acontece en las situaciones de sistemas de comunicación ampliados modifica esos patrones de excitación, gestionando otras rutinas y promoviendo la desaparición de otras. Las necesidades de excitación están ahí, los estímulos para ello pueden estar prescritos y bajo control, sistema de comunicación sujeto a ciertos sistemas de información, pero también sucede que aparezcan nuevos estímulos, o sistemas de información alternos que afectan lo prescrito y programado.

La excitación es una poderosa vivencia de reconfiguración situacional de los sistemas de información prescriptivos. Todos los sistemas sociales buscan en alguna forma, sistemas de información, controlar el metabolismo de la excitación, en ocasiones lo logran a tal punto que terminan promoviendo ciclos entrópicos de vida social, muerte vital del deseo, y en otras ocasiones fracasan, y la excitación se dispara en forma destructiva, la emoción del desorden y el caos.

La excitación es clave en reproducción de la vida social, peligroso es su debilitamiento, peligrosa es su expansión sin control alguno. Todo está en el sutil equilibrio entre los sistemas de información y los sistemas de comunicación y su relación dinámica. Y claro, la Ingeniería en Comunicación Social puede resolver los extremos, o producirlos, puede promover el equilibrio o desestabilizarlo.

El caso de la vivencia y la experiencia estéticas es complementario y distinto al de la excitación. La vivencia estética supone un desequilibrio en la reproducción de los sistemas de comunicación prescritos por ciertos sistemas de información. A diferencia de la excitación no es necesaria en un sentido energético y vital básico, pero es un detonador de una complejidad que tiene un efecto reorganizador en el sistema de vida social en general, su potencial reconstructivo es muy significativo. $Y$ esta es la cualidad básica de la vivencia estética en su camino a tomar la forma de una experiencia estética, la complejidad.

La excitación es simple y poderosa, reordena por estallido energético, como una erupción, el resultado en casos extremos es un desastre, un escenario resultante por completo imprevisible. La vivencia estética es compleja e intensa, reordena también con una onda energética, pero reorganizando en forma ordenada a la percepción. La excitación es repetitiva, tiende a reproducirse como un sistema de información que busca y obtiene siempre el mismo resultado, de ahí su degradación por la rutina.

En cambio la vivencia estética es siempre distinta por la reorganización perceptual. Se parecen más de lo que en este momento se presenta. Una es visceral, la otra es cognitiva superior. Son como la misma vivencia pero en un orden y escala distintas. La excitación viene de abajo, es terrenal, la vivencia estética aspira a la totalidad, es cósmica. Ambas son situacionales, la primaria es prescribible con ciertas dificultades, la segunda es más difícil de promover y gestionar. La primera puede ser modelizada, la segunda menos. La Ingeniería en Comunicación Social tiene retos importantes en la observación y la comprensión de ambas. Quizás en ellas está la llave de la evolución humana. La música es un tremendo instrumento para su configuración, una tecnología social adecuada para explorarlas y desarrollarlas. Como en el caso del rock y el jazz. 
4. El rock y el jazz en el mundo contemporáneo mexicano

Esta cuarta parte propone una hipótesis de trabajo sobre el desarrollo del rock y del jazz en el mundo contemporáneo, en particular en México. El rock ha tenido una presencia más popular en general por ser más simple y directo, el jazz es más complejo, más denso para la experiencia estética. En México el rock ha tenido una presencia que se agota en mucho en una situación de excitación extrema y contestataria simple.

El jazz no se ha desarrollado en México al requerir una formación compleja, y una articulación más amplia con la vida social. Es un lugar común que para tocar el rock se requiere poca formación musical académica, o ninguna, y para tocar el jazz se necesita mucha formación académica y práctica. Un breve apunte sobre la historia de ambas formas musicales en México puede servir para identificar las trayectorias y las tendencias de su presencia, y con ello dar las bases para la formulación de una hipótesis sobre su impacto en la cultura mexicana.

El rock aparece en México a través de los medios de difusión entre el final de los años cincuenta y principios de los años sesenta. A lo largo de aquellos primeros años de la presencia del fenómeno en el país son importantes de entrada dos factores, la emergencia de la cultura popular industrial global a través de los medios de difusión masiva, y las condiciones para su recepción en el contexto nacional y regional. Después de la segunda guerra mundial el país se articula en forma intensa con la industria de los Estados Unidos, incluyendo a la industria cultural.

La televisión y el cine son un fenómeno indiscutible que afecta a la cultura nacionalista con connotaciones agrarias de la primera parte del siglo XX. El efecto es sobre todo en las ciudades, y en particular en las grandes ciudades. Por otra parte está la configuración de clase y de consumo. La cultura popular norteamericana está más cerca de las clases medias urbanas en emergencia, ellas son las que se conforman en el nicho consumidor de lo producido en EE. UU., desde la ropa, los cosméticos, la comida industrializada, hasta los discos, las películas y las series de televisión.

Este fenómeno tiene sus antecedentes en las décadas anteriores, para finales de los años cincuenta las clases altas y medias urbanas ya son consumidores confirmados consuetudinarios de las marcas y los productos importados. El país está articulado al mercado del norte y en el caso de la cultura industrial el fenómeno va en aumento. El rock llega al país en esas condiciones, existe una curiosidad de los jóvenes de las clases altas en formar parte del movimiento universal occidental del consumo de la nueva cultura juvenil. No todos los jóvenes responden en forma similar, pero hay una presencia del mercado musical suficiente como indicador para afirmar que el negocio de lo juvenil está en marcha también en México.

Por otra parte hay regiones que no son afectadas más que en forma marginal, la cultura tradicional popular del campo y la música mexicana proveniente de la industria del disco y la radio nacional están vigentes. El país está en emergencia hacia la cultura global producida en EE. UU. , pero el fenómeno no es general y extenso, aunque si guarda una tendencia hacia la expansión cultural general. Este es un gran tema de estudio para entender a México en la segunda parte del siglo $\mathrm{XX}$.

Durante los años sesenta la industria cultural nacional promueve un tipo de producción del rock peculiar, copias de lo que sucede en EE. UU., el consumo de este tipo de rock se complementa con lo que viene directo de aquel país, y que se escucha en la radio y en discos. Para los ochenta y los noventa, después de la cruda del sesenta y ocho y la casi prohibición del rock por el efecto aglutinador y sentido contestatario de su estética y lírica, el fenómeno adquiere un segundo aire, se convierte en un fenómeno nacional y el mercado se extiende a todas partes, incorporando a la población juvenil urbana en general al movimiento juvenil de consumo de las formas y los estilos provenientes del norte.

Para finales del siglo $X X$ y a lo largo del siglo $X X I$ el rock ya es parte de la cultura popular nacional, conviviendo con diversas formas musicales y culturales desarrolladas dentro de la cultura pop ya globalizada. Hoy el rock ha pasado de ser sólo algo que representaba una ruptura y una diferencia marcada de la cultura mexicana tradicional, para ser parte de una nueva cultura mexicana popular urbana también nacional. El consumo y reproducción de su estética es un fenómeno general juvenil y más allá, después de varias décadas de su presencia en el territorio nacional.

Al jazz le sucede algo parecido que al rock en su presencia y desarrollo primario en México, también viene del norte, tiene un efecto significativo en la ciudad de México, al igual que el rock, pero es aún más selectivo y elitista, se asocia en principio a 
requiere de una atención y de una empatía compleja con la situación musical y con el contexto que articula. El rock requiere una atención mínima, su empatía es simple y en ocasiones no es necesaria, es sólo consumo individual ardiendo en un momento de excitación. Son formas de música distintas, son formas de vivencia en vivo distintas, son formas de construcción del sentido y la empatía distintas.

Sólo para aclarar y enfocar el asunto central en el tema de la experiencia estética es necesario un comentario sobre el campo de la experiencia estética en ambas formas musicales. El punto es que estando ligadas en la situación particular en la configuración del sistema de comunicación, la vivencia estética del músico y la del público no son iguales.

Los investigadores del asunto desde la perspectiva cognitiva apuntan que la música estimula diversas partes del cerebro, y dependiendo del tipo de música pueden ser más o menos, en este sentido el jazz es más rico que el rock. Pero algo que también señalan los especialistas es que la vivencia musical del que toca un instrumento y la del que sólo escucha música es distinta, la del músico es mucho más rica que la del que sólo escucha.

El punto aquí es que el sistema de comunicación del concierto de música es distinto al sistema de comunicación de escuchar la música grabada, y que la configuración de la situación en concierto es diversa según la afinidad y empatía entre el público y los músicos. Por un lado músicos y público viven la situación musical de diversa forma, y por otra parte la situación de concierto es distinta de la escucha de música grabad, y por otra parte lo anterior es distinto si estamos en sistema de comunicación jazz o un sistema de comunicación rock.

Todo esto establece un programa de observación y estudio sobre el tema de la vivencia y la posterior construcción de la experiencia estética en el jazz o el rock como sistemas de información y sistemas de comunicación. Necesitamos configuraciones generales más o menos estabilizadas para un apunte comparativo. Los tipos posible generales pueden ordenarse sobre el gradiente cultura popalta cultura. En el caso de la cultura pop se trata de grandes públicos y una articulación situacional en el sistema de comunicación de un público que no es conocedor, sólo gozador o escucha casi distraído. $Y$ en complemento la música de este punto del gradiente es algo que se acerca a un lugar común, una forma musical que no requiere ningún esfuerzo para ser reconocida y apreciada, parecida a formas ya muy conocidas, standars, y construida en una configuración musical simplificada.

En el caso de la alta cultura se trata de públicos más selectivos y de dimensiones mucho menores al gran público. La música en este punto del gradiente es más compleja en su composición e interpretación, tiende a ser música original, que exige del músico y del ensamble mucha concentración y atención, gran calidad de interpretación y conocimiento en forma y ejecución mucho mayor que en la música pop.

El público de este tipo de música corresponde en buena parte a toda esta complejidad, es más sofisticado, más culto, un escucha atento y concentrado en lo que sucede, con más conocimiento musical que el término medio, mucho más exigente con lo que escucha y auto exigente con su condición de escuchar. Las situaciones de concierto en uno y otro caso pueden ser extremas, en el caso de la música pop el público espera diversión y motivo para excitación rápida y continua. En el caso de la música de alta cultura el público espera entretenimiento superior, un gozo de las formas en donde escuchar la música está en el centro, no la diversión y la alteridad social.

El rock y el jazz tienen ubicación dentro de este gradiente, pero el rock tiene una tendencia hacia la experiencia de la cultura pop, y el jazz hacia la alta cultura. Esto expresa que ambos sistemas de información y comunicación pueden ser muy variados y diversos, pero las trayectorias han ido posicionando al rock más cerca de la cultura pop y la experiencia estética por excitación, y al jazz más cerca de la alta cultura y la experiencia estética por emoción compleja.

A partir de este esquema lo que sigue tiene dos caminos principales. Por una parte identificar qué es lo que está detrás de estas trayectorias básicas, y por otra parte identificar cuáles son las trayectorias secundarias, y cómo es que se ha configurado todo este espacio tiempo de la vida estética de una y otra forma musical en la vida social y cultural.

Primera nota. El jazz es más fino y por eso no es popular. El rock es más burdo y por eso es más popular. Esto parece tener un marco de referencia histórico y social que lo comprueba. El rock como fenómeno popular tiene como coartada que las baladas y las formas musicales melódicas han construido un público masivo, menos exigente en lo estructural musical, más exigente respecto a lo sencillo y simple, lo cercano. 
El jazz como fenómeno musical tiene una exigencia mayor en la composición original, y una exigencia mayor en la gramática de esa composición original, su público típico especializado aplaude ese esfuerzo y subestima lo común y convencional. El músico de rock está más en consonancia con la configuración cultural y musical del público masivo popular. El público del jazz está más cerca de la configuración cultural y musical del músico exigente y de gran formación.

Segunda nota. El rock también tiene una versión de alta cultura, y el jazz también tiene una versión cercana a la música popular. Hay versiones del rock que requieren una gramática musical más rica, sus pretensiones son mayores que en el rock común. Ese es el caso del rock progresivo, que a lo largo de las décadas se ha ido posicionando como un tipo distinto de rock, más complejo para públicos más sofisticados.

El tema de mercado también es factor, pero el crecimiento y desarrollo de los públicos también es factor. Por otra parte hay un jazz que se considerada degradado, el tipo de jazz para acompañar las compras en el supermercado, o en la sala de espera del dentista o en el elevador. El elemento melódico suele ser el punto constructivo básico. Es tan sutil y diluido que puede ser escuchado sin poner atención en absoluto.

El jazz de los standars de bar y cantina también tiene la función de música de fondo tocada por músicos en vivo. Este tipo de jazz le gusta a casi todo mundo, aunque no lo escuche a menudo y no lo pueda identificar con claridad, es algo para no escuchar con atención. La dimensión de alta cultura del rock también adquiere el formato de lo inaudible, entre más ruidoso e incomprensible mejor, rock con vocación de marginalidad, hecho en apariencia para los pocos, duros y obscuros, que termina tomando la forma de una categoría especial del mercado. El jazz popular se convierte en un lugar común, en una cápsula predecible y reducida a una vivencia sin tensión. La fórmula queda representada en los discos y conciertos de ciertos saxofonistas y pianistas que incluso adquieren cierta fama masiva y popular.

Tercera nota. El rock en su vocación de situación de excitación, emoción concentrada, puede ser más o menos complejo. En general el rock es menos complejo, el jazz es más complejo. La paradoja del rock, que en su estilo más directo y duro, el heavy metal, adquiere su mayor configuración concentrada de provocación de la excitación, estalla en diversidad en el nicho de su popularidad de connotaciones adolescentes.

La gramática del heavy metal no es compleja, pero la cantidad de rolas que se han producido bajo su forma es hoy una multitud. Esto lleva el análisis a otro nivel, el rock se ha complejizado por su desarrollo demográfico y popular. Sigue siendo inferior al jazz en gramática, pero la ejecución es energéticamente muy exigente, lo que cumple el requisito de la excitación, pero también complejiza el modelo de la ejecución y de la situación del sistema de comunicación, el concierto de rock en vivo es muy exigente en ejecución y espectacularidad.

El jazz por otra parte se mueve hacia sus fronteras en todas direcciones, al tener menos fidelidades a formas standars, y una vocación musical de gran curiosidad, toma vuelo en las fusiones y los experimentos musicales de todo tipo. El jazz llega en estos momentos a una inmensa cantidad de formas musicales. El rock se mezcla menos aún con cierto perfil de fusión, el jazz es muy promiscuo. El punto de diferencia está en la vocación musical, que en el jazz es mucho mayor. El rock está más cargado de sensualidad y espectáculo, de lugares comunes y rituales. El jazz está más cargado de musicalidad y exploración experimental.

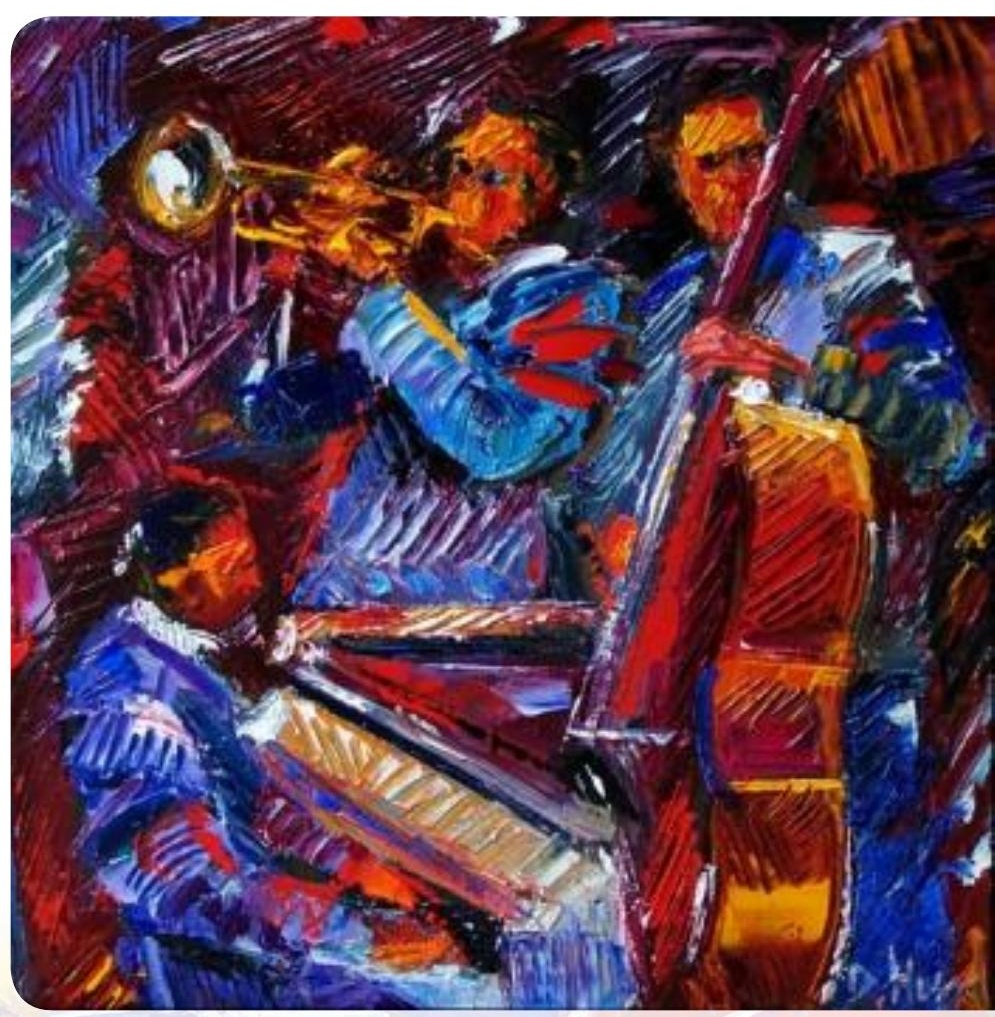




\section{Y la experiencia estética?}

Ambos sistemas de comunicación social son gestores de experiencia estética. En la figura de lo musical el jazz es más íntimo, más experiencia musical, y desde el interior conecta a los participantes en un rito en que la música articula desde el interior, promueve una comunión social una octava más arriba que el rock, conectando a muchos desde la música en un sentido cercano a la meditación.

El rock es más exterior, extrovertido, en ocasiones es sólo eso y nada más. Conecta en forma más sensorial, con una necesidad mayor de la escenificación situacional. Pero estas son sólo generalizaciones, que aunque tienen referentes reales, son simplificaciones de un marco de vivencia y experiencia estética que la música como telón general es rica en enacción.

El rock se configura en la vivencia de un grito visceral y energético, el jazz en la vivencia de un momento trascendente consciente. Uno es más terrestre, el otro más cósmico. Ambos necesitan de la otra parte, y así sucede. La mejor historia sobre la experiencia estética del rock y el jazz es la historia de su relación, la vivencia conmovedora desde la sensualidad que rompe las represiones sentimentales y emocionales, en complemento con la vivencia conmovedora desde la emoción enriquecida que conecta lo cercano con lo lejano aumentando la empatía. Quizás esta historia sea sólo el relato de cómo la naturaleza se comunica con la civilización en el mundo contemporáneo.

\section{Bibliografía}

AGUSTíN, José (1972) La Nueva Música Clásica, Ediciones INJUVE, México.

ARANA, Federico Arana (1985) Guaraches de ante azul. Historia del rock mexicano. Tomos 1, 2, 3 y 4, Posada, México.

AYMES, Roberto (2009) Panorama del Jazz en México Durante el siglo XX, LUZAM, Cuernavaca.

BIANCHI, Filippo y Pier Paolo Pitacco (2012) 101 Microlecciones de Jazz, Editorial Océano, Barcelona.

BERENDT, Joachim E. (1994) El Jazz. De Nueva Orleans al Jazz Rock, Fondo de Cultura Económica, Bogotá.

BLACKMORE, Susan (2000) La máquina de los memes, Paidós, Barcelona.
BODEN, Margaret (1994) La mente creativa, Editorial Gedisa, Barcelona.

BREA, José Luis (2008) El tercer umbral. Estatuto de las prácticas artísticas en la era del capitalismo cultural. Editorial CENDEAC, Murcia.

BYRNE, David (2014) Cómo funciona la Música, Editorial Sexto Piso, Ciudad de México.

CALABIA, Enrique, Santiago Cano, Lorenzo Rodríguez y Julián Ruiz (2008) Historia del Rock and Roll, El País. Madrid.

CALABRESE , Omar (1987) El lenguaje del arte, Paidós, Barcelona.

CASACUBERTA, David (2003) Creación colectiva, Gedisa, Barcelona.

CHO, Adrian (2010) The Jazz Process. Collaboration, Innovation, and Agility, Pearson Education, Boston.

DE LA CALLE, Luis y Luis Rubio (2010) Clasemedieros. Pobre no más, desarrollado aún no, Centro de Investigación para el desarrollo, México.

DELLA VOLPE, G. (1963) Crítica del gusto, Seix Barral, Barcelona.

DELANNOY, Luc (2001) ¡Caliente! Una historia del jazz latino, Fondo de Cultura Económica, Ciudad de México.

DELANNOY, Luc (2012) Convergencias. Encuentros y desencuentros en el jazz latino, Fondo de Cultura Económica, Ciudad de México.

DERBEZ, Alain (2001) El Jazz en México. Datos para esta historia. Fondo de Cultura Económica, Ciudad de México.

DUMAZEDIER, J. (1964) Hacia una civilización del ocio, Estela, Barcelona.

ECO, Umberto (1978) Tratado de Semiótica general, Nueva imagen-Lumen, México.

ELIAS, Norbert y Eric Dunning (1995) Deporte y ocio en el proceso de la civilización, Fondo de Cultura Económica, México.

GALBRAITH, J. K. (1992) La cultura de la satisfacción, Ariel, Madrid.

GALINDO Cáceres Jesús (2006) Cibercultura. Un mundo emergente y una nueva mirada. CNCAInstituto mexiquense de la cultura, Toluca. 
GALINDO Cáceres, Luis Jesús (2011) Ingeniería en Comunicación Social y Promoción Cultural. Sobre Cultura, Cibercultura y Redes Sociales, Homo Sapiens, Universidad Nacional del Rosario, Benemérita Universidad Autónoma de Puebla, Rosario.

GALINDO Cáceres, Luis Jesús (2014) Ingeniería en Comunicación Social. Hacia un Programa General, Instituto de Ciencias de Gobierno y Desarrollo Estratégico-Benemérita Universidad Autónoma de Puebla, Puebla.

GARDNER , Howard (1993) Arte, mente y cerebro, Paidós, Barcelona.

GREGORY, Hugo (1999) Un Siglo de Pop. Cien años de música que cambiaron al mundo, Blume, Barcelona.

HOUD É, Olivier, et al. (2003) Diccionario de Ciencias Cognitivas, Amorrortu editores, Buenos Aires.

GIOIA, Ted (2002) Historia del Jazz, Fondo de Cultura Económica, Ciudad de México.

LEADBEATER, Charles (2010) Cloud Culture. The future of global cultural relations. Counterpoint, London.

MALACARA Palacios, Antonio. (2005) Catálogo casi razonado del Jazz en México, Angelito, Ciudad de México.

MALACARA Palacio, Antonio (2001) Catálogo Subjetivo y Segregacionista del Rock Mexicano, CONACULTA/FONCA, México.

MALACARA Palacio, Antonio (Coordinador) (2008) Viaje al fondo del Jazz. Coloquio-Memoria, Delegación Coyoacán-Universidad Autónoma de Baja California-Benemérita Universidad Autónoma de Puebla, Ciudad de México.

MARSALIS, Wynton (2012) Jazz. Cómo la música puede cambiar tu vida, Espasa Libros, Barcelona.

MARTEL, Frédéric (2010) Cultura Mainstream. Cómo nacen los fenómenos de masas, Taurus, México.

MASLO W, Abraham (1990) La personalidad creadora, Kairos, Barcelona.

MATURANA R., Humberto (1997) Emociones y lenguaje en educación y política. Dolmen, Santiago.

MAZZOLA, Guerino y Paul B. Cherlin (2009) Flow,
Gesture, and Spaces in Free Jazz. Towards a Theory of Collaboration, Springer-Verlag, Berlin Heidelberg.

McDONALD, D. (1969) La industria de la cultura, Alberto Corazón, Madrid.

MERLEAU-PONTY, Maurice (1994) Fenomenología de la Percepción, Ediciones Península, Barcelona.

MIEGE, B., P. Pajon y J. Salaün (1986) L'industrialisation de l'audiovisuel, Res Babel, París.

MOLES, Abraham (1976) Teoría de la información y percepción estética, Júcar, Madrid.

MUGGIATI, Roberto (1974) Rock, el grito y el mito: la música pop como forma de comunicación y contracultura, Siglo XXI editores, México.

PAKMAN, Marcelo (compiador) (1997)Construcciones de la experiencia humana (dos volúmenes), Gedisa, Barcelona.

VARELA, Francisco (1990) Conocer, Gedisa, Barcelona.

WAGENSBER G, Jorge (1994) Ideas sobre la complejidad del mundo, Tusquets, Barcelona.

YÚDICE, G. (2002) El recurso de la cultura. Usos de la cultura en la era global, Gedisa, Barcelona.

\section{Para citar este artículo:}

GALINDO Cáceres, Jesús (2015). INGENIERÍA EN COMUNICACIÓN SOCIAL DE LA EXPERIENCIA ESTÉTICA DEL ROCK Y EL JAZZ. Explorando la configuración de la vida contemporánea a través de la música. Revista Luciérnaga/Comunicación, Año 7, N13. Facultad de Comunicación AudiovisualPolitécnico Colombiano Jaime Isaza CadavidPCJIC \& Facultad de Ciencias de la Comunicación - Universidad Autónoma de San Luis Potosí- UASLP. México. ISSN 2027- 1557. Págs. 1-12. 